\title{
Influencia del tipo de bujía en la evaluación del comportamiento y emisiones en un motor de encendido provocado
}

\section{(Influence of the type of spark plug in the evaluation of the performance and emissions caused in an ignition engine)}

\author{
Gabriel Serpa ${ }^{1}$, Xavier Zumbaํ․ Francisco Montalvo ${ }^{1}$, Daysi Baño ${ }^{1}$, Jorge Martinez ${ }^{1}$, Iván Zambrano ${ }^{1}$
}

\begin{abstract}
Resumen
La presente investigación se realiza en la ciudad de Cuenca a 2500 m.s.n.m. y analiza la influencia del tipo de bujía utilizada en un motor de encendido provocado (MEP) en las prestaciones mecánicas del motor, sus emisiones contaminantes y el consumo específico del combustible. Se utilizan seis tipos de bujías disponibles en el mercado nacional, las mismas que presentan diferentes características de material, grado térmico, apertura y números del electrodo. El comportamiento mecánico del motor y el consumo específico de combustible se evalúan mediante un banco de pruebas en un dinamómetro; las emisiones contaminantes se miden bajo el protocolo de prueba estática establecido por la NTE INEN 2203 y con una prueba de ruta de $11 \mathrm{~km}$ en zona urbana y carretera a base del ciclo de conducción NEDC (New European Driving Cycle). En el estudio se obtiene un incremento del 16 \% en el rendimiento del motor para un tipo específico de bujiá, conjuntamente con la disminución en la emisión de gases contaminantes, menor consumo de combustible y mejor comportamiento del motor en frío, sin sacrificar el torque y la potencia del vehículo de prueba. Además, se aprecia un ahorro en el mantenimiento del motor.
\end{abstract}

\section{Palabras clave}

Motor de encendido provocado; emisiones contaminantes; torque y potencia; bujías.

\begin{abstract}
This research was made in Cuenca at 2500 meters above sea level (masl), and it studied the influence of the use of different types of spark plugs in a spark-ignition engine on the mechanical performance of the engine, the pollutant emissions, and fuel consumption. Six types of spark plugs that are available in the national market are made of different materials, have different thermal properties, and have different electrode openings. In addition, the mechanical performance of the engines and their fuel consumption were evaluated through dynamometer testing. Moreover, the polluting emissions were measured under the static test protocol established by the NTE INEN 2203 and with an 11 $\mathrm{km}$ route test in urban areas and on highway based on the New European Driving Cycle. The research showed that a specific spark plug increases $16 \%$ of the engine performance due to not only some factors such as less pollution, better cold behavior, lower fuel consumption, but also maintains the torque and power of the vehicle. In addition, this spark plug generates savings on maintenance and annual fuel consumption.
\end{abstract}

\section{Keywords}

Spark-ignition engine, spark plugs, pollutant emissions; torque and power.

\section{Introducción}

Esta investigación busca determinar la influencia del tipo de bujía utilizado en las prestaciones mecánicas y en las emisiones contaminantes en un vehículo con motor de encendido provocado (MEP) con inyección electrónica utilizando seis tipos de bujías diferentes, considerando los altos índices de contaminación ambiental en la ciudad de Cuenca como resultado del incremento en la demanda de vehículos; según la AEADE se estima un crecimiento del parque auto-

1 Escuela Politécnica Nacional, Quito-Ecuador (\{gabriel.serpa, xavier.zumba, francisco.montalvom, daysi.banom, jorge.martinez, ivan.zambrano\}@epn.edu.ec). 
motor del 13 \% anual. Con base en los estudios realizados por la Unidad de Tránsito Municipal de la ciudad de Cuenca; el incremento del parque automotor ha elevado los índices de ruido y gases contaminantes en el ambiente. La municipalidad de Cuenca con la finalidad de mejorar la calidad del aire ha implementado controles de emisiones y de estado mecánico a todos los vehículos matriculados en la ciudad a partir de 2008.

Actualmente, las emisiones contaminantes han sido un tema cuyo impacto ambiental se ha venido estudiando bajo diferentes circunstancias, por ejemplo, Antamba et al. (2016) evalúa las emisiones de un vehículo en toda la comunidad andina en donde determinan que el tipo de gasolina utilizado y el lugar en donde trabaja el motor hace que las emisiones varíen; asimismo Llanes, Rocha, Peralta y Leguísamo, (2018), estudian a las emisiones vehiculares específicamente en la ciudad de Quito a 2800 m.s.n.m., verificando que los factores de emisión de un vehículo serán diferentes en condiciones de altura con respecto al nivel del mar.

En este sentido, se han buscado diferentes alternativas que permitan la reducción de los gases contaminantes, sin dejar de lado la disminución del consumo de combustible. En un estudio realizado por Guzman et al. (2018) se utilizan mezclas de gasolina extra (87 octanos) y gasolina super (92 octanos), obteniendo una disminución de emisiones contaminantes con una mezcla determinada; asimismo el uso de tecnología GDI en el motor permite disminuir las emisiones de contaminación (Krishna, Mallikarjuna y Davinder, 2016). Sin embargo, dentro de la cámara de un motor no solo la inyección del combustible afecta directamente al proceso de combustión, sino también las características que presenta la bujía utilizada, por esta razón su correcta aplicación puede garantizar el desempeño óptimo del motor; sus características de diseño como el espacio de la brecha, tamaño y número del electrodo, y la configuración de la punta influyen en el rendimiento del motor con la menor emanación de gases contaminantes (Burgett, Leptich y Sangwan, 2007). Según Saw y Mallikarjuna (2017) la ubicación del inyector de combustible y de la bujía influyen en gran medida en la mezcla aire-combustible, la combustión, el rendimiento y características de emisión. La combinación de la bujía ubicada en la parte central de la cámara de combustión y el inyector de combustible a un lado produce una mejor combustión y rendimiento en el motor. Costa y Allocca (2011) también afirman que la orientación del inyector de combustible influye de manera significativa en la formación de la mezcla.

Sjeri'c, Taritaš y Kozarac (2017) mediante una simulación confirman que el uso de del electrodo de iridio en la bujía mejora la combustión y la estabilidad sobre el rango de operación analizado del motor, con una mejora máxima de 13.5\% en carga parcial y baja velocidad del motor. También establecen que la geometría de la bujía tiene potencial para mejorar la estabilidad del motor y la economía de combustible. Yorita et al. (2007) mostraron que el centro fino y el electrodo de tierra de la bujía permiten una mejor inflamabilidad y mejora la eficiencia de combustión. Con esta investigación concuerdan Lee y Boehler (2005) quienes probaron el rendimiento de un motor con tres diferentes tipos de bujías principales: una bujía estándar J-gap con un electrodo central de $2.5 \mathrm{~mm}$, una bujía J-gap con un electrodo central de $0.6 \mathrm{~mm}$ y una bujía de descarga de superficie con un electrodo central de $0.4 \mathrm{~mm}$ y cuatro electrodos de tierra; en donde observaron que la bujía J-gap con el electrodo central fino tenía el rendimiento del motor más estable y menor consumo de combustible. Es decir, una bujía con finos electrodos de centro y de tierra producen una menor combustión variación con una reducción de aproximadamente $3.1 \%$ en CO y $2.4 \%$ en el consumo de combustible en comparación con la chispa normal (Ahmed y Rehim, 2012). 
Burgett et al. (1972) coinciden con Tawfik, Bao y Xu (2017) en que el efecto de la brecha de la bujía es dominante para tener una mezcla pobre, rica o estequiométrica. Pero además, Tawfik et al. (2017) concluyen que la potencia del motor aumenta ligeramente y la combustión se vuelve más estable a medida que aumenta el espacio de la bujía. Con la brecha máxima de la bujía, el motor produce emisiones mínimas de hidrocarburos. Sin embargo, las emisiones de NOx se incrementan a medida que la brecha de la bujía se agranda debido a la temperatura más alta acompañada con el aumento de la velocidad de la llama y la presión en el cilindro.

Por lo tanto, la evaluación de emisiones, el comportamiento del motor y el consumo de combustible para diferentes bujías es una alternativa que mediante su experimentación puede plantear soluciones muy prácticas que disminuyan las emisiones contaminantes sin comprometer el desempeño del motor.

\section{Metodología}

Los ensayos realizados en el estudio determinan los valores de algunas emisiones contaminantes como: monóxido de carbono $(\mathrm{CO})$, hidrocarburos $(\mathrm{HC})$, dióxido de carbono $\left(\mathrm{CO}_{2}\right)$ y el factor lambda. Además, se analiza el comportamiento del motor obteniendo información del ancho de pulso de la inyección, el avance de encendido, consumo de combustible, el torque y la potencia. Estos ensayos se realizan mediante pruebas estáticas y pruebas dinámicas. Las pruebas dinámicas se efectúan en ruta y en un dinamómetro.

Antes de llevar a cabo las pruebas mencionadas se desinstaló el catalizador del sistema de evacuación de gases, evitando de esta manera que los gases contaminantes emitidos por el motor sean tratados por este dispositivo, analizando así los valores netos de los gases sin el post-tratamiento respectivo.

El desarrollo de las pruebas hace referencia a la norma NTE INEN 2203 y ciclo al europeo de conducción NEDC, realizando tres mediciones en cada ensayo.

\subsection{Equipos utilizados}

El estudio se realiza con un vehículo de marca Chevrolet, modelo Grand Vitara de año de fabricación 2014, sus características técnicas se detallan en la Tabla 1.

Tabla 1. Características técnicas del vehículo

\begin{tabular}{|l|l|}
\hline Cilindrada [cc] & 1996 \\
\hline Alimentación & MPFI \\
\hline n. ${ }^{\circ}$ de cilindros & 4 en línea \\
\hline n. de válvulas & 16 \\
\hline Potencia [KW arpm] & 94.7 @6000 \\
\hline Par [Nm @rpm] & 173,6 @4301 \\
\hline Transmisión & Mecánica 4x2 \\
\hline Velocidades & 5 - reversa \\
\hline Capacidad de carga $[\mathrm{kg}]$ & 650 \\
\hline
\end{tabular}

La medición de emisiones se realiza mediante un analizador de gases NGA 6000 QROTECH mostrado en la Figura 1, y sus datos técnicos se indican en la Tabla 2. 
Tabla 2. Especificaciones del analizador de gasees NGA 6000.

\begin{tabular}{|l|l|l|l|l|l|}
\hline & \multicolumn{1}{|c|}{ CO } & \multicolumn{1}{c|}{ CO $_{2}$} & \multicolumn{1}{c|}{$\mathbf{H C}$} & \multicolumn{1}{c|}{$\mathrm{O}_{2}$} & \multicolumn{1}{c|}{ Lambda } \\
\hline Rango de medición & $0.00-9.99 \%$ & $0.0-20 \%$ & $0-9999 \mathrm{ppm}$ & $0.00-25 \%$ & $0-2$ \\
\hline Resolución & $0.01 \%$ & $0.1 \%$ & $1 \mathrm{ppm}$ & $0.01 \%$ & $0.001 \%$ \\
\hline
\end{tabular}

Figura 1. Analizador de gases NGA 6000 QROTECH

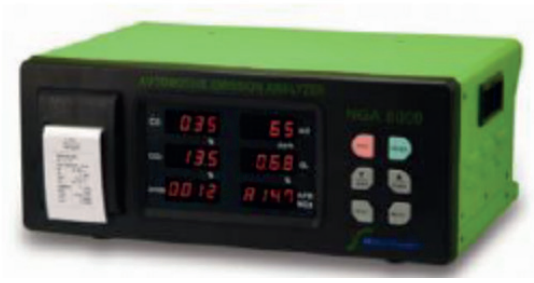

Una parte de las pruebas dinámicas se realizan en un dinamómetro marca MAHA LPS 3000 mostrado en la Figura 2, en él se puede determinar la potencia y torque máximos del motor para cada tipo de bujía que se utilice en el vehículo. El equipo cumple con las normas internacionales: SAE J1349 o JIS D1001, EWG 80/1269, DIN 70020,ISO 1585.

Figura 2. Dinamómetro LPS 3000
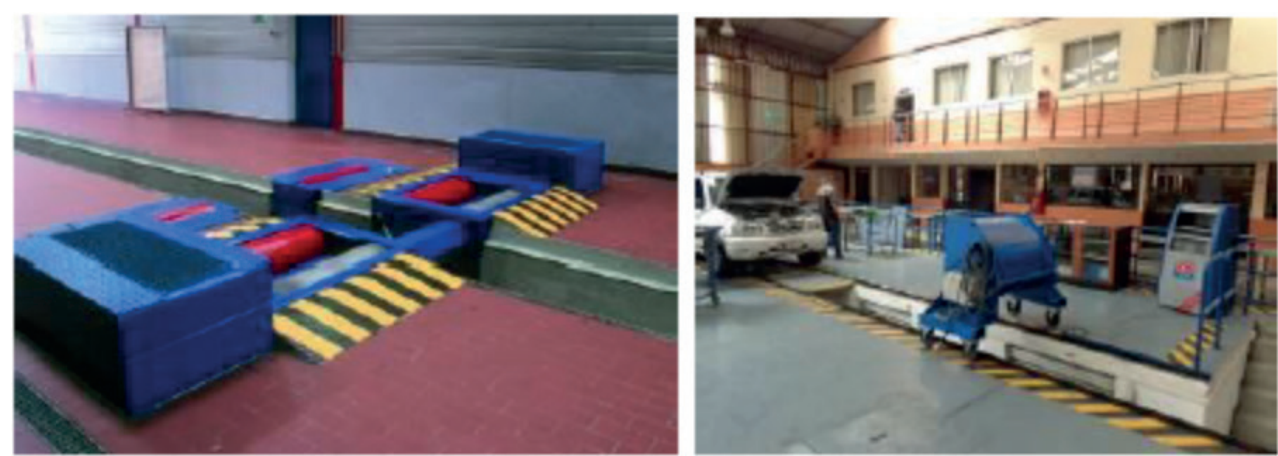

Durante las pruebas estáticas y dinámicas se monitorea el ancho de pulso de inyección y el avance de encendido con un scanner automotriz marca Carman Scan Lite.

Los tipos de bujias utilizados basándose en la disponibilidad el mercado nacional presenta diferentes características de material, grado térmico, apertura y geometría del electrodo y se han identificado con una numeración, en la Tabla 3 se detallan las características de cada bujía utilizada.

Tabla 3. Características de las bujías utilizada.

\begin{tabular}{|l|l|l|l|}
\hline \multicolumn{1}{|c|}{ Bujía } & \multicolumn{1}{|c|}{ Material del electrodo } & \multicolumn{1}{c|}{ Codificación } & \multicolumn{1}{c|}{ Grado térmico } \\
\hline Tipo 1 & aleación de cobre-níquel & BKR6E-11 & medio \\
\hline Tipo 2 & aleación de cobre-níquel & MFR3LS & frío \\
\hline Tipo 3 & Iridio & IK20 & medio \\
\hline Tipo 4 & cuatro electrodos, aleación de cobre-níquel & UXF79 & frío \\
\hline Tipo 5 & platino & FR8DPX & caliente \\
\hline Tipo 6 & aleación de cobre-níquel & BKR5E-11 & caliente \\
\hline
\end{tabular}




\subsection{Protocolo de pruebas, prueba estática}

La prueba estática se realiza una vez que el motor haya conseguido su temperatura normal de funcionamiento en el motor, en marcha mínima (ralentî) y a 2500 rpm para cada tipo de bujía en estudio. Se midieron cuatro tipos de gases contaminantes que son: hidrocarburos (HC), monóxido de carbono (CO), dióxido de carbono $\left(\mathrm{CO}_{2}\right)$ y oxígeno $\left(\mathrm{O}_{2}\right)$, además se registró el valor del factor lambda, ancho de pulso de inyección y avance de encendido. Esta prueba se realizó considerando el protocolo establecido en la norma INEN 2 203, mismo que se describe a continuación:

- Ubicar el vehículo en el punto de partida.

- Instalar los equipos de instrumentación en el vehículo.

- Encender el motor.

- Estabilizar en ralentí a la temperatura normal de funcionamiento.

- Acelerar y estabilizar el régimen del motor a 2500 × 300 rpm.

- Tomar datos

- Estabilizar el motor en ralentí (Tiempo de espera $30 \mathrm{~s}$ aproximadamente).

- Tomar datos.

- Apagar el motor.

- Cambiar el tipo de bujía.

\subsection{Protocolo de Pruebas, prueba dinámica}

En el estudio se realizaron dos tipos de pruebas dinámicas para cada bujía, una prueba en ruta que consiste en la medición de gases y consumo de combustible mediante un ciclo de conducción establecida por la NEDC; y una prueba que evalúa el comportamiento mecánico del motor en un dinamómetro.

La prueba en ruta establecida por la NEDC considera un tramo de 11 km con una duración de 1180 segundos en donde se realiza cuatro repeticiones del ciclo urbano y un ciclo en carretera de forma consecutiva, en donde la primera repetición evalúa el comportamiento en frío del motor. El modo de conducción para el ciclo urbano se muestra en la Figura 3, este ciclo se repite cuatro veces, y luego se realiza el ciclo de carretera que se indica en la Figura 4.

Figura 3. Ciclo NEDC urbano EPA. (2013). Testing and measuring emissions (en línea)

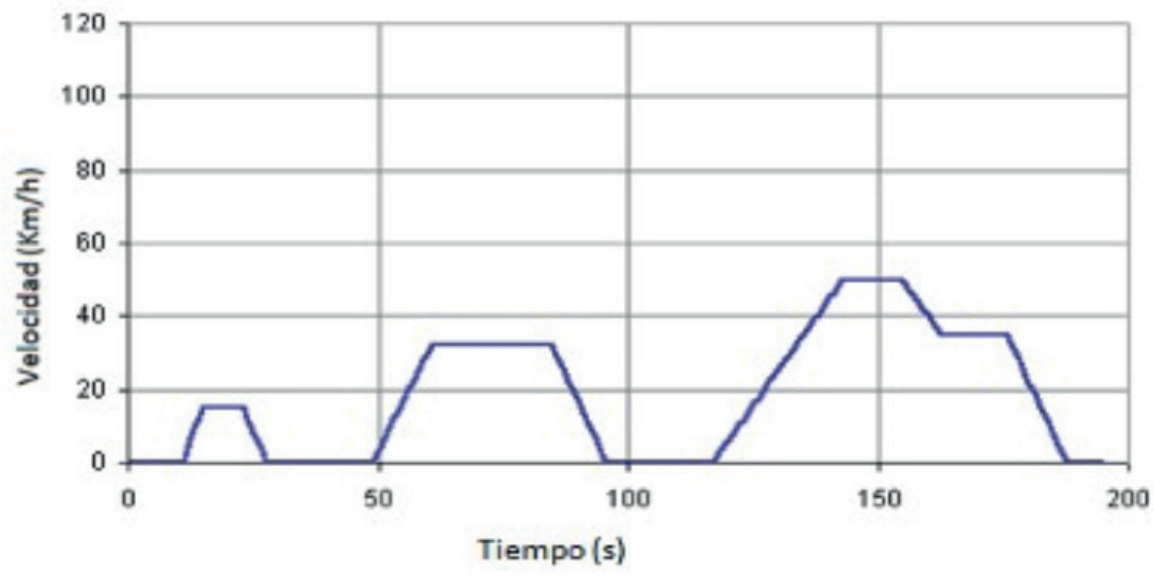


Figura 4. Ciclo NEDC en carretera EPA. (2013). Testing and measuring emissions (en línea)

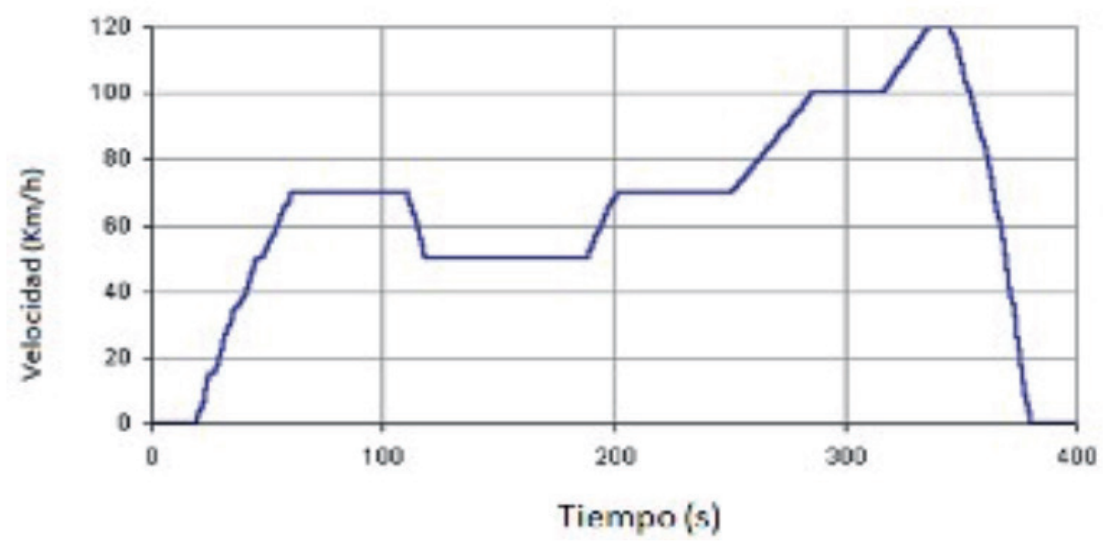

Para ajustar el ciclo de conducción europeo NEDC a una prueba de ruta es importante considerar las siguientes condiciones de ruta y ambientales (SAE, 2002)

- Ausencia de lluvia o niebla

- Carretera en buen estado: seca, limpia y lisa (Asfalto o Concreto).

- Pendiente menor al $0.5 \%$.

- Temperatura ambiente $\left(20^{\circ} \mathrm{C}-30{ }^{\circ} \mathrm{C}\right)$.

- Tramo recto de $11 \mathrm{~km}$.

- Tramos sin cruces peligrosos.

- Carretera.

- Velocidad promedio del viento menor a $24 \mathrm{~km} / \mathrm{h}$ o con picos mayores a $32 \mathrm{~km} / \mathrm{h}$.

El protocolo de pruebas realizado para el ciclo de conducción NEDC se detalla a continuación:

- Ubicar el vehículo en el punto de partida.

- Mantener en reposo al vehículo durante 6 horas.

- Constatar condiciones ambientales e iniciales.

- Iniciar con el ciclo urbano (el primero evalúa el comportamiento en frío del motor)

- Repetir el ciclo urbano tres veces más.

- Llevar a cabo el ciclo de carretera.

El dinamómetro se utilizó para determinar la potencia y torque máximos del motor para los diferentes tipos de bujías a ensayar.

- Posicionar el vehículo sobre los rodillos del dinamómetro.

- Instalar el ventilador frente al vehículo.

- Conectar los instrumentos de medición de rpm y temperatura del motor.

- Ingresar los datos técnicos del vehículo y el tipo de prueba a realizar.

- Encender el vehículo y esperar hasta su temperatura normal de funcionamiento.

- Verificar las rpm del motor con las que se registra en el equipo.

- Encender el ventilador.

- Acelerar el vehículo hasta la cuarta marcha (relación de trasmisión 1:1), sin exceder los $50 \mathrm{~km} / \mathrm{h}$. 
- Iniciar la prueba de medición.

- Presionar completamente el acelerador, hasta que el equipo indique que se presione el embrague, y por último un mensaje indica que se acelere nuevamente hasta que se termine la medición.

- Apagar el ventilador.

- Generar informe en la pantalla del banco de pruebas.

- Cambiar de bujía.

- Realizar nuevamente los ensayos de medición.

\section{Resultados y discusión}

Luego de realizar todas las pruebas requeridas para cada tipo de bujía, en la ciudad de Cuenca, en la Tabla 4 se presentan los resultados de las pruebas estáticas a 2500 rpm.

Tabla 4. Prueba estática (2500 rpm)

\begin{tabular}{|l|c|c|c|c|c|c|c|}
\hline Bujía & $\begin{array}{c}\mathrm{HC} \\
{[\mathrm{ppm}]}\end{array}$ & $\begin{array}{c}\mathrm{CO} \\
{[\%]}\end{array}$ & $\begin{array}{c}\mathrm{CO}_{2} \\
{[\%]}\end{array}$ & $\begin{array}{c}\mathrm{O}_{2} \\
{[\%]}\end{array}$ & Lambda & $\begin{array}{c}\text { Ancho de pulso } \\
{[\mathrm{msec}]}\end{array}$ & $\begin{array}{c}\text { Avance de encendido } \\
{[\text { grados] }}\end{array}$ \\
\hline Tipo 1 & 73 & 0.42 & 13.2 & 1.64 & 1.062 & 2.91 & 38.17 \\
\hline Tipo 2 & 74 & 0.39 & 13.3 & 1.83 & 1.073 & 3.21 & 38.33 \\
\hline Tipo 3 & 65 & 0.51 & 13.5 & 1.69 & 1.074 & 3.27 & 38.17 \\
\hline Tipo 4 & 78 & 0.41 & 14.1 & 1.02 & 1.031 & 3.35 & 38.17 \\
\hline Tipo 5 & 63 & 0.52 & 13.5 & 1.8 & 1.065 & 3.14 & 38.33 \\
\hline Tipo 6 & 74 & 0.45 & 13.5 & 1.81 & 1.069 & 3.17 & 38.33 \\
\hline
\end{tabular}

Con la bujía tipo 5, las emisiones de hidrocarburos y el ancho de pulso son más bajos que con los otros tipos de bujías. La bujía tipo 2 ayuda al motor a emitir la menor cantidad de monóxido de carbono.

Los resultados de la prueba estática en ralentí se indican en la Tabla 5.

Tabla 5. Prueba estática (ralentí)

\begin{tabular}{|l|c|c|c|c|c|c|c|}
\hline Bujía & $\begin{array}{c}\mathrm{HC} \\
{[\mathbf{p p m}]}\end{array}$ & $\begin{array}{c}\mathbf{C O} \\
{[\%]}\end{array}$ & $\begin{array}{c}\mathrm{CO}_{2} \\
{[\%]}\end{array}$ & $\begin{array}{c}\mathrm{O}_{2} \\
{[\%]}\end{array}$ & Lambda & $\begin{array}{c}\text { Ancho de pulso } \\
{[\mathbf{m s e c}]}\end{array}$ & $\begin{array}{c}\text { Avance de encendido } \\
\text { [grados] }\end{array}$ \\
\hline Tipo 1 & 163 & 0.38 & 13.1 & 1.69 & 1.065 & 2.38 & 14 \\
\hline Tipo 2 & 170 & 0.3 & 13.1 & 2.29 & 1.098 & 2.47 & 15 \\
\hline Tipo 3 & 179 & 0.4 & 13.4 & 2.27 & 1,091 & 2.44 & 14 \\
\hline Tipo 4 & 222 & 0.31 & 14.6 & 0.86 & 1.021 & 2,36 & 14 \\
\hline Tipo 5 & 152 & 0.48 & 12.9 & 2.06 & 1.082 & 2,41 & 13.5 \\
\hline Tipo 6 & 174 & 0.31 & 13.4 & 2.05 & 1.084 & 2.34 & 14 \\
\hline
\end{tabular}

Se observa que a excepción de la bujía tipo 4, con todas las bujías se tienen valores menores a los límites que establece la norma NTE INEN 2204, (\%CO <1, HC $<200$ ppm). La bujía tipo 5 presenta los valores más bajos en cuanto a emisiones y para todos los casos, la combustión se realiza con una mezcla pobre.

En la Tabla 6 se presentan los valores de la prueba dinámica en ruta. 
Tabla 6. Prueba dinámica, ciclo NED

\begin{tabular}{|c|c|c|c|c|c|c|c|c|}
\hline & \multicolumn{2}{|c|}{$\begin{array}{c}\text { 1er Ciclo urbano } \\
\text { comportamiento } \\
\text { en frío) }\end{array}$} & \multicolumn{7}{|c|}{3 ciclos urbanos + 1 ciclo de carretera } & \\
\hline Bujía & $\begin{array}{c}\text { HC } \\
{[p p m]}\end{array}$ & $\begin{array}{c}\text { CO } \\
{[\%]}\end{array}$ & $\begin{array}{c}\text { HC } \\
{[\mathrm{ppm}]}\end{array}$ & $\begin{array}{c}\text { CO } \\
{[\%]}\end{array}$ & $\begin{array}{c}\mathbf{C O}_{2} \\
{[\%]}\end{array}$ & $\begin{array}{c}\mathbf{O}_{2} \\
{[\%]}\end{array}$ & Lambda & $\begin{array}{c}\text { Rendimiento } \\
{[\mathrm{km} / \text { litro] }}\end{array}$ \\
\hline Tipo 1 & 220.35 & 0.69 & 175.52 & 0.5 & 12.81 & 2.23 & 1.09 & 9.4 \\
\hline Tipo 2 & 362.66 & 0.55 & 203.62 & 0.39 & 12.72 & 2.98 & 1.14 & 7.95 \\
\hline Tipo 3 & 245.11 & 0.85 & 174.68 & 0.59 & 13.36 & 2.77 & 1.12 & 8.9 \\
\hline Tipo 4 & 254.3 & 1.36 & 222,29 & 0,57 & 14.22 & 1.49 & 1.05 & 9.25 \\
\hline Tipo 5 & 179.53 & 0.65 & 150.24 & 0.59 & 16.66 & 2.69 & 1.12 & 8.1 \\
\hline Tipo 6 & 177.88 & 0.55 & 164.67 & 0.67 & 13.23 & 2,51 & 1.11 & 9.7 \\
\hline
\end{tabular}

La bujía tipo 6 presenta menores valores de emisiones cuando el motor se encuentra frío y un mayor rendimiento de kilómetros por litro de combustible, sin embargo, la bujía tipo 5 presenta un mejor desempeño en todo el ciclo NEDC.

La evaluación del comportamiento del motor se realizó mediante la medición de la potencia máxima y el torque máximo a través de una carga simulada en un dinamómetro. La potencia obtenida para cada bujía se indica en la Tabla 7.

Tabla 7. Torque máximo para cada bujía @4500 rpm

\begin{tabular}{|l|c|c|c|}
\hline Bujía & $\begin{array}{c}\text { Potencia } \\
{[\mathbf{K W}]}\end{array}$ & $\begin{array}{c}\text { Torque máx. } \\
{[\mathbf{N m}]}\end{array}$ & $\begin{array}{c}\text { Consumo } \\
{[\mathbf{g} / \mathbf{K W h}]}\end{array}$ \\
\hline Tipo 1 & 70.66 & 149.93 & 201.79 \\
\hline Tipo 2 & 71.16 & 150.93 & 211.68 \\
\hline Tipo 3 & 70.93 & 150.51 & 213.76 \\
\hline Tipo 4 & 70.19 & 148.95 & 209.06 \\
\hline Tipo 5 & 70.19 & 148.94 & 217.8 \\
\hline Tipo 6 & 71.33 & 151.36 & \\
\hline
\end{tabular}

Considerando que hay una diferencia menor al $2 \%$, se aprecia que con la bujía tipo 6 se consigue el torque máximo, parámetro que relaciona el consumo máximo de combustible.

La variación de la potencia para cada bujía ensayada se indica en la Tabla 8, con la bujía tipo 2 se obtiene la potencia y consumo máximo.

Tabla 8. Potencia máxima para cada bujía @6000 rpm

\begin{tabular}{|l|c|c|c|}
\hline & $\begin{array}{c}\text { Potencia máx. } \\
{[\mathbf{K W}]}\end{array}$ & $\begin{array}{c}\text { Torque } \\
{[\mathbf{N m}]}\end{array}$ & $\begin{array}{c}\text { Consumo } \\
{[\mathbf{g} / \mathbf{K W h}]}\end{array}$ \\
\hline Tipo 1 & 87.83 & 139.77 & 216.22 \\
\hline Tipo 2 & 90.22 & 143.59 & 267.94 \\
\hline Tipo 3 & 88.69 & 141.16 & 258.17 \\
\hline Tipo 4 & 87.58 & 139.39 & 220.45 \\
\hline Tipo 5 & 87.8 & 139.73 & 234.36 \\
\hline Tipo 6 & 88.93 & 141.53 & 210.98 \\
\hline
\end{tabular}

En forma general, considerando todas las pruebas realizadas se demuestra que cada bujía ofrece diversos comportamientos en función de sus características como se muestra en las Figuras 5,6,7,8,9,10. 
Figura 5. HC [ppm] prueba estática

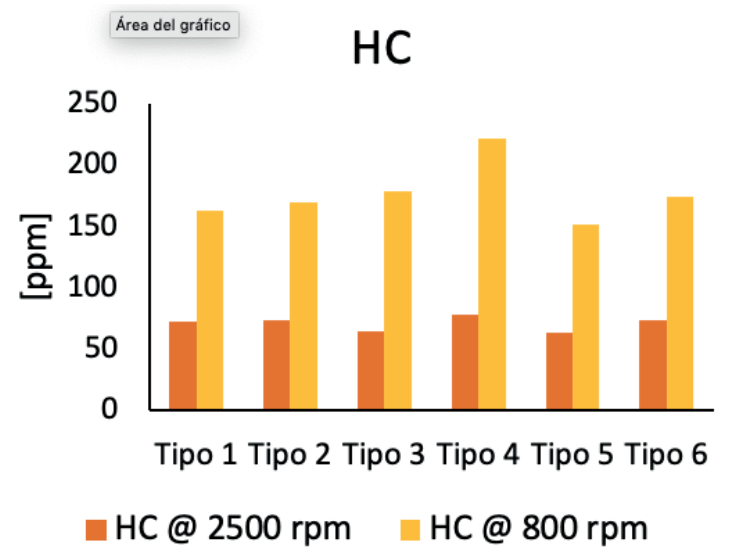

Figura 7. HC [ppm] prueba dinámica

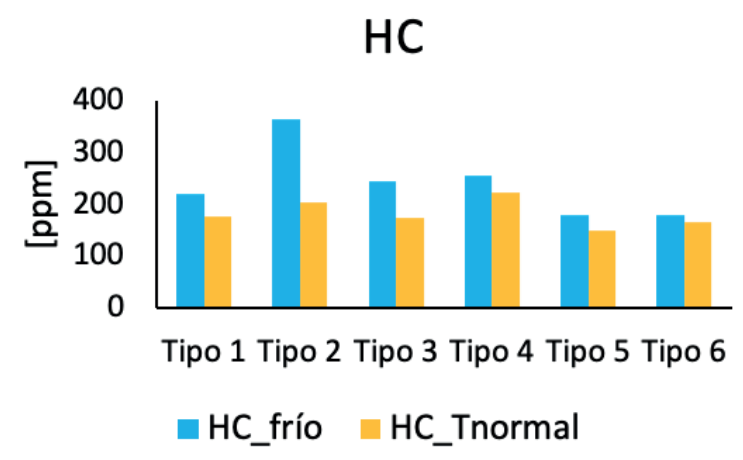

Figura 9. Torque y Potencia máxima

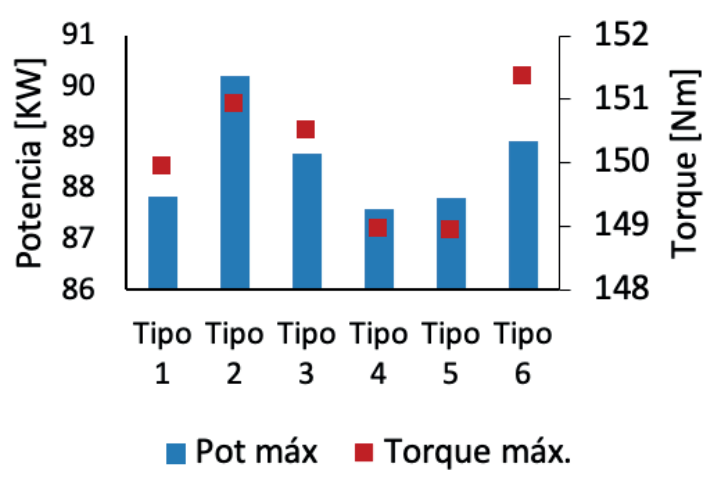

Figura 6. CO [\%] prueba estática

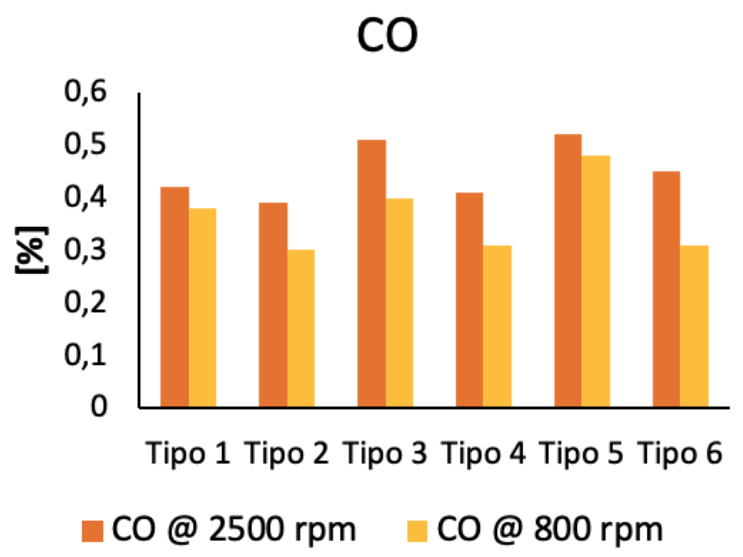

Figura 8. CO [\%] prueba dinámica

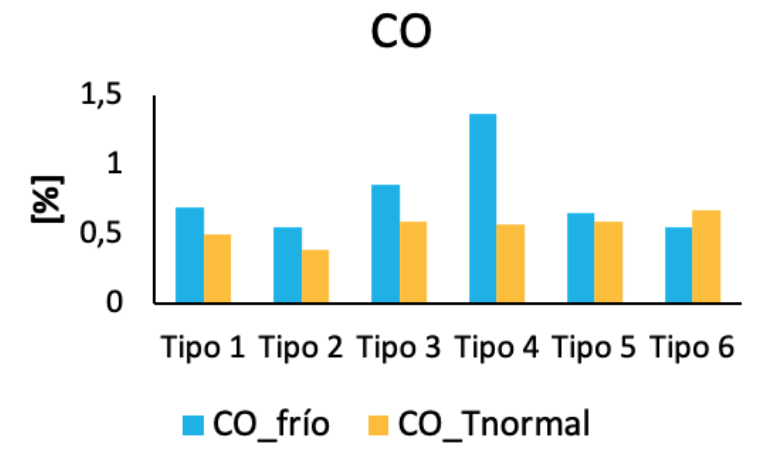

Figura 10. Rendimiento de combustible

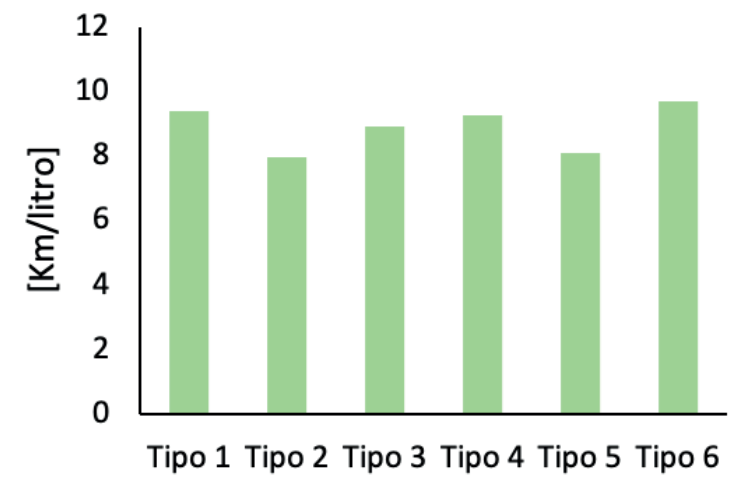


Con la bujía tipo 5 y tipo 6 se expide menor cantidad de gases contaminantes, se presenta un mejor comportamiento en frío, un mayor rendimiento de combustible sin sacrificar las prestaciones mecánicas del motor de torque y potencia; estas bujías corresponden a un grado térmico caliente, y tienen características similares a excepción del material del electrodo. Tomando en cuenta el consumo de combustible y la prueba de potencia se considera que la bujía tipo 6 es la recomendada para su uso en el vehículo de prueba.

\section{Conclusiones y recomendaciones}

Cada tipo de bujía utilizada en un motor de combustión interna corresponde a un comportamiento diferente en sus prestaciones mecánicas y emisiones contaminantes, por esta razón es importante determinar cuál es el tipo de bujía mas adecuado para obtener un mejor desempeño y menor consumo de combustible.

Al analizar el resultado de las pruebas estáticas y dinámicas se observa que con una bujía de un solo electrodo, grado térmico caliente se puede mejorar en un $16 \%$ el desempeño del motor, es decir, que con este tipo de bujía se expide menor cantidad de gases contaminantes, se tiene mejor comportamiento en frío, mayor rendimiento de combustible sin afectar significadamente el torque y potencia del vehículo.

El uso de una bujía con un grado térmico menor (bujía caliente), puede ayudar a mantener una temperatura adecuada de funcionamiento en la cámara de combustión en las condiciones ambientales y de funcionamiento en una ciudad a 2500 m.s.n.m., lo que se refleja en la disminución de emisión de los hidrocarburos.

La brecha del electrodo o luz del mismo es un parámetro que influye en las emisiones contaminantes; sería importante establecer la luz mas adecuada del electrodo para diferentes alturas sobre el nivel del mar.

Evaluar el comportamiento mecánico y emisiones de otros motores con el tipo de bujía que ha presentado el mejor desempeño, así se aumentará el número de la muestra y se podrá generalizar los datos obtenidos.

\section{Bibliografía}

Antamba,J., Reyes, G., y Granja, M. (2016). Estudio comparativo de gases contaminantes en un vehículo M1, utilizando gasolina de la Comunidad Andina. Enfoque UTE, V.7- 3, 110-119.

Abdel-Rehim, A. A. (2013). Impact of spark plug number of ground electrodes on engine stability. Ain Shams Engineering Journal, 4(2), 307-316. https://doi.org/10.1016/j.asej.2012.09.006

Badawy, T., Bao, X. C., y Xu, H. (2017). Impact of spark plug gap on flame kernel propagation and engine performance. Applied Energy, 191, 311-327. https://doi.org/10.1016/j.apenergy.2017.01.059

Burgett R, Leptich J, Sangwan K. Measuring the effect of spark plug and ignition system design on engine performance. SAE technical paper 720007; 1972. http://dx.doi.org/10.4271/720007

Costa M. U. S. y Allocca L. (2011). Numerical study of the mixture formation process in a Fourstroke GDI Eengine for two-wheel applications Simulation Modelling Practice and Theory, 19 (2011), 1212-1226.

Craver R., Podiak R., y Miller R. Spark plug design factors and their effect on engine performance. SAE technical paper 700081; 1970. http://dx.doi.org/10.4271/700081

EPA. (2013). Testing and measuring emissions (en línea). U.S. Recuperado de: www3.epa.gov/nvfel/testing/dynamometer.htm. (accedido el 21/03/2018). 
Guzmán, A., Cueva, E., Peralvo, A., Revelo, M., y Armas, A. (2018). Estudio del rendimiento dinámico de un motor Otto al utilizar mezclas de dos tipos de gasolinas: "Extra " y "Súper "; Enfoque UTE, 9(4), 208-220.

Hori, T., Shibata, M., Okabe, S., y Hashizume, K. (2003). Super ignition spark plug with fine center and ground electrodes. SAE paper 2003-01-0404.

Krishna, S., Mallikarjuna, J. M. y Davinder, K. (2016). Effect of Engine Parameters on In-Cylinder Flows in a Two-Stroke Gasoline Direct Injection Engine. Applied Energy 176, 282-294.

Lee, Y. G., y Boehler, J. T. (2005). Flame kernel development and its effects on engine performance with various spark plug electrode configurations. SAE Technical Paper 2005-01-1133, Society of Automotive Engineers, Detroit.

Llanes, E., Rocha, J., Peralta, D., y Leguísamo, J. (2018). Evaluación de emisiones de gases en un vehículo liviano a gasolina en condiciones de altura. Caso de estudio Quito, Ecuador. Enfoque UTE, 9(2), 149-158.

Nishio, K., Oshima, T., Ogura, H. (1994). A study on spark plug electrode shape. Int J Veh Des, 15, 119-30.

Norma Técnica Ecuatoriana NTE INEN 2 203:2000. Gestión Ambiental. Aire. Vehículos Automotores. Determinación de la concentración de emisiones de escape en condiciones de marcha mínima o

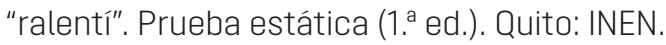

Norma Técnica Ecuatoriana NTE INEN 2 204:2002. Gestión Ambiental. Aire. Vehículos Automotores. Límites permitidos de emisiones producidas por fuentes móviles terrestres de gasolina (1. ${ }^{a}$ ed.). Quito, Ecuador: INEN.

Osamura, H. (2000). "Development of long life and high ignitability iridium spark plug. Seoul 2000 FISITA world automotive congress, Paper number F2000A144, Korea.

Saw, O. P., \& Mallikarjuna, J. M. (2017). Effect of spark plug and fuel injector location on mixture stratification in a GDI engine-A CFD analysis. IOP Conference Series: Materials Science and Engineering (vol. 243). https://doi.org/10.1088/1757-899X/243/1/012025

Shimanokami, Y., Matsubara, Y., Suzuki, T., y Matsutani, W. (2004). Development of high ignitability with small size spark plug. SAE paper 2004-01-0987.

Sjerić, M., Taritaš, I., y Kozarac, D. (2017). Effect of Spark Plug Geometry on the Cyclic Combustion Variability and Fuel Consumption of Gasoline Engines. Journal of Energy Engineering, 143(6). https:// doi.org/10.1061/(ASCE)EY.1943-7897.0000492

Yamaguchi, T., Nakamura, S., y Oshima, T. (1987). Spark plug and its electrode configuration. Google Patents; 1987

Yorita, H., Okabe, S. I., Ishiguro, H., y Shibata, M. (2007). Ignition simulation and visualization for spark plug electrode design. SAE Technical Paper 2007-01-0940, Society of Automotive Engineers, Detroit. 